\title{
New breakthrough CRISPR/Cas9 biotechnology of genome editing is a powerful tool for improvement of agricultural crops
}

Kershanskaya O.I.*, Nelidova D.S., Esenbaeva G.L. Mukiyanova G.S., Nelidov S.N. Institute of Plant Biology and Biotechnology, Almaty, Kazakhstan

*e-mail:gen_o.kersh@mail.ru

The technology, a genome-editing tool called CRISPR/Cas9, revolutionized the life sciences when it appeared on the market in 2013. It is now proving useful in the plant science community as a powerful tool for the improvement of agricultural crops. CRISPR/Cas9 system is a simple, inexpensive and versatile tool for gene/genome editing, resulting in which has become known as the 'CRISPR craze'. The multitude of functions that can be performed with CRISPR/Cas9 make it a molecular tool that will open new opportunities in the complicated world of plant-pathogen interactions and help design durable crop resistance to pathogens. Our goal is to create elite barley cultivars, resistant to viral diseases, in Kazakhstan. Using the CRISPR/Cas9 tool editing of eIF4E gene that conferred resistance to multiple viruses has been successfully engineered in 5 commercial Kazakhstan barley cultivars. Elite crops cultivars will benefit farmers and the local economy. Generally, the CRISPR/Cas9 system for plant genome editing is a breakthrough technology in breeding - prospect for creation of elite high yielding crops that could be exempt from GM classification. 